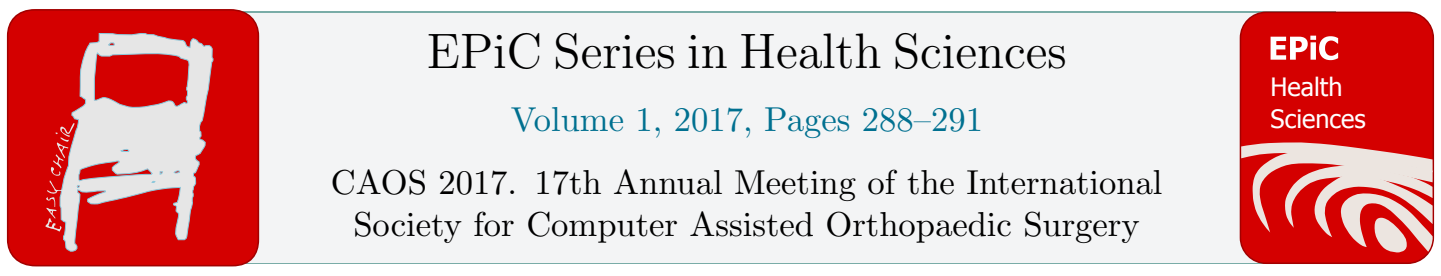

\title{
Robotic-Arm Assisted Total Knee Arthroplasty Demonstrated Soft Tissue Protection
}

\author{
Emily L. Hampp PhD ${ }^{1}$, Manoshi Bhowmik-Stoker $\mathrm{PhD}^{1}$, Laura Y. Scholl MSc ${ }^{1}$, Jason K. Otto $\mathrm{PhD}^{1}$, \\ David J. Jacofsky MD² and Michael A. Mont $\mathrm{MD}^{3}$ \\ ${ }^{1}$ Stryker, Mahwah, NJ, USA \\ ${ }^{2}$ The Core Institute, Phoenix, AZ, USA \\ ${ }^{3}$ Cleveland Clinic, Cleveland, OH, USA \\ emily.hampp@stryker.com, manoshi.bhowmik-stoker@stryker.com, \\ laura.sholl@stryker.com, jason.otto@stryker.com, \\ david.jacofsky@thecoreinstitute.com, montm@ccf.org
}

\begin{abstract}
Total knee arthroplasty (TKA) typically addresses end-stage osteoarthritis. While TKA procedures have demonstrated clinical success, occasionally intraoperative complications can occur. This cadaver study examined the potential benefits of soft tissue protection in robotic-arm assisted TKA (RATKA). Six cadaver knees were prepared using RATKA by a single surgeon from a high-volume TKA joint center with no former clinical robotic experience, and compared to seven manually performed cases as a control. The presence of soft tissue disruption was assessed by having the surgeon perform visual evaluation and palpation of the medial collateral ligament (MCL), lateral collateral ligament (LCL), posterior cruciate ligament (PCL), and the patellar ligament after the procedures. The amount of tibial subluxation and patellar eversion was recorded for each case.

For all RATKA cases, there was no visible evidence of disruption of any of the ligaments. All RATKA cases were successfully left with a bone island on the tibial plateau, which protected the PCL. Tibial subluxation and patellar eversion were not required for visualization. In two of the seven MTKA cases, there was slight disruption noted of the PCL, although this did not lead to any apparent change in the functional integrity of the ligament. All MTKA cases required tibial subluxation and patellar eversion to achieve optimal visualization.

Aspects of soft tissue protection were noted in this cadaver study for RATKA. Standard retraction techniques during cutting are recommended. This is the first study to have soft tissue injury parameters assessed for RATKA, and may serve as a platform for future studies.
\end{abstract}




\section{Introduction}

While manual total knee arthroplasty (MTKA) procedures have demonstrated clinical success in terms of pain relief and survivorship, occasionally intraoperative complications can occur [1]. Medial/lateral collateral, or posterior cruciate ligament injury, instability, extensor mechanism disruption, and tibio- and patella-femoral dislocation are among a few of the adverse outcomes prevalently ranked by The Knee Society in the U.S., some of which are associated with intraoperative complications and defined ICD-9 codes [1-7]. Robotic-arm assisted TKA (RATKA) provides a surgeon the ability to three dimensionally plan a TKA and use intraoperative visual, auditory, and tactile feedback to ensure that only the desired bone cuts are made. The potential benefits of soft tissue protection in these surgeries need to be further evaluated. The purpose of this cadaver study was to assess the a) integrity of various knee soft tissue structures (medial collateral ligament (MCL), lateral collateral ligament (LCL), posterior cruciate ligament (PCL), and the patellar ligament), as well as b) the need for tibial subluxation and patellar eversion in RATKA procedures.

\section{Materials and Methods}

Six cadaver knees were prepared using RATKA by a single surgeon from a high-volume TKA joint center with no former clinical robotic experience. These were compared to seven manually performed cases as a control. The presence of soft tissue disruption was assessed by having an experienced surgeon perform visual evaluation and palpation of the PCL, MCL, LCL, and the patellar ligament after the procedures. In addition, leg pose and retraction was documented during all bone resections. The amount of tibial subluxation and patellar eversion was recorded for each case.

\section{Results}

For all RATKA cases, there was no visible evidence of disruption of any of the ligaments. All RATKA cases were successfully left with a bone island on the tibial plateau, which protected the PCL (example cadaveric specimen and corresponding planned tibial resection shown in Figure 1). Tibial subluxation and patellar eversion were not required for visualization. In two of the seven MTKA cases, there was slight disruption noted of the PCL, although this did not lead to any apparent change in the functional integrity of the ligament. All MTKA cases required tibial subluxation and patellar eversion to achieve optimal visualization.

\section{Discussion and Conclusion}

Several aspects of soft tissue protection were noted during the study. During bone resections, the tibia in RATKA procedures did not require subluxation, which may reduce ligament stretching. Further, patellar eversion may lead to increased length-of-stay (LOS), increased complication rate, and decreased post-operative range of motion [8, 9]. Arnout et al. [8] performed a prospective, randomized, double blinded study of 60 TKAs performed using medial parapatellar approach, who either had patellar eversion or patellar subluxation. There was a significantly better improvement of both passive and active range of motion (ROM) in patellar subluxation, when compared to patellar eversion cohort at 1 year follow-up. In a meta-analysis of five randomized controlled trials assessing patellar eversion during primary TKA, Yang et al. [9] demonstrated that patellar non-eversion was associated with shorter mean 
length-of-stay, decreased complication rate, but longer tourniquet time. Potential patient benefits for short-term recovery and decreased morbidity due to reduced operative complications should be studied in a clinical setting. Since RATKA uses a stereotactic boundary to constrain the sawblade, which is generated based on the implant size, shape and plan, and does not have the ability to track the patient's soft tissue structures, standard retraction techniques during cutting are recommended. The present study utilized cadavers instead of live patients, which may not allow for results to be translated in vivo. Additionally, assessment did not include more detailed evaluation of ligaments (histological, biochemical, etc.). However, this is the first study to have soft tissue injury parameters assessed for RATKA, and may serve as a platform for future studies.
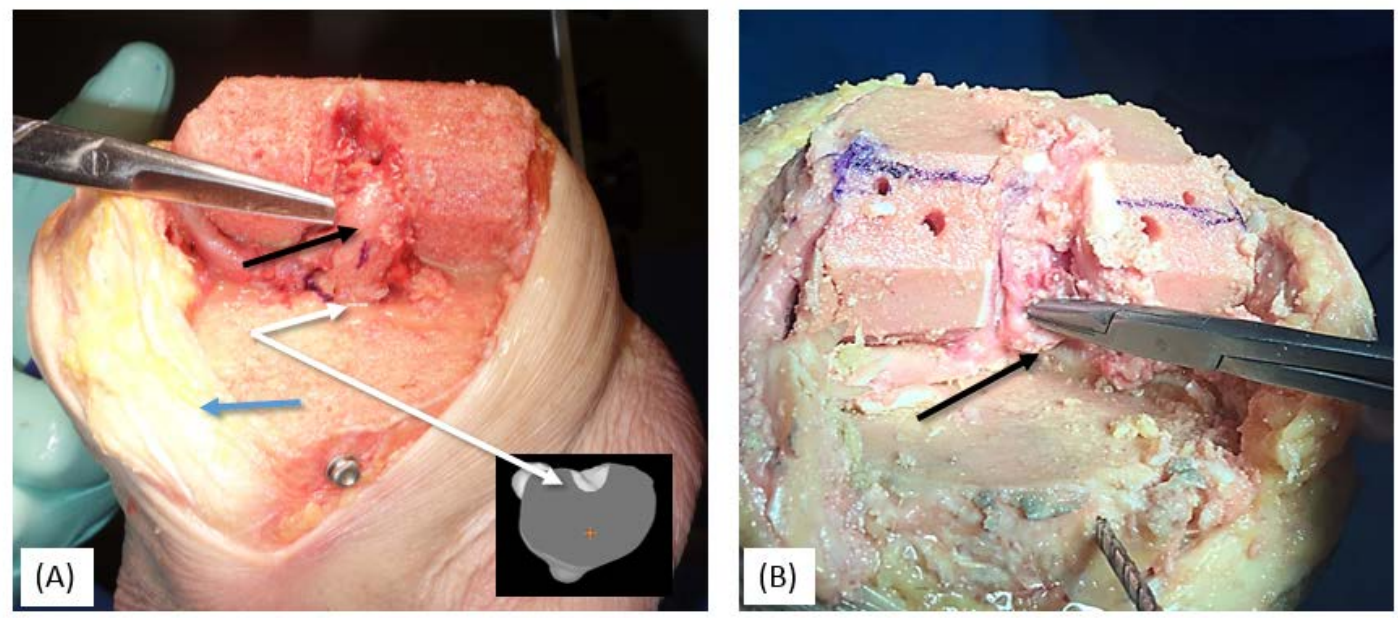

Figure 1: A) A robotic-arm assisted TKA with bone island preparation in front of PCL and corresponding tibial view from Implant Planning page (showing actual boney anatomy, with remaining bone island). B) Manually performed TKA with arrow pointing to PCL, with no bone island preparation. Black arrow points to A) intact PCL in the RATKA and B) minor fray of PCL in the MTKA. White arrow outlines bone island. Blue arrow points to intact patellar ligament.

\section{References}

[1] Wijdicks CA, Griffith CJ, Johansen S, Engebretsen L, LaPrade RF. Injuries to the medial collateral ligament and associated medial structures of the knee. J Bone Joint Surg Am, 92(5), pp. 1266-80, 2010. [2] Leopold SS, McStay C, Klafeta K, Jacobs JJ, Berger RA, Rosenberg AG. Primary repair of intraoperative disruption of the medical collateral ligament during total knee arthroplasty. J Bone Joint Surg Am, 83-A(1), pp. 86-91, 2001.

[3] Siqueira M, Haller K, Mulder A, Goldblum A, Klika A, Barsoum W. Outcomes of Medial Collateral Ligament Injuries during Total Knee Arthroplasty. J Knee Surg, 29(1), pp. 68-73, 2014.

[4] McNabb DC, Kim RH, Springer BD. Instability after total knee arthroplasty. J Knee Surg, 28(2), pp. 97-104, 2015.

[5] Bates MD, Springer BD. Extensor Mechanism Disruption After Total Knee Arthroplasty. J Am Acad Orthop Surg, 23(2), pp. 95-106, 2015.

[6] Barrack RL, Butler RA, Valenzuela R. Extensor mechanism disruption after total knee arthroplasty: when the unthinkable happens. Orthopedics, 25(9), pp. 981-2, 2002. 
[7] Lynch AF, Rorabeck CH, Bourne RB. Extensor mechanism complications following total knee arthroplasty. J Arthroplasty, 2(2), pp. 135-40, 1987.

[8] Arnout N, Victor J, Cleppe H, Soenen M, Van Damme G, Bellemans J. Avoidance of patellar eversion improves range of motion after total knee replacement: a prospective randomized study. Knee Surgery, Sport Traumatol Arthrosc, 17(10), pp. 1206-10, 2009.

[9] Yang G, Huang W, Xie W, Liu Z, Zheng M, Hu Y, et al. Patellar non-eversion in primary TKA reduces the complication rate. Knee Surgery, Sport Traumatol Arthrosc, 24(3), pp. 921-30. 2016.

\section{Disclosures}

Emily L. Hampp, Manoshi Bhowmik-Stoker, Laura Y. Scholl, and Jason K. Otto are Stryker employees. David J. Jacofsky and Michael A. Mont are consultants for Stryker. 